\title{
PREDICTORS OF IRINOTECAN TOXICITY AND EFFICACY IN TREATMENT OF METASTATIC COLORECTAL CANCER
}

\begin{abstract}
Adam Paulik, Jiř̌i Grim, Stanislav Filip
Charles University in Prague, Faculty of Medicine and University Hospital Hradec Králové, Czech Republic: Department of Oncology and Radiotherapy

Summary: The colorectal cancer ranks high among the malignant tumours in incidence and mortality and irinotecan is standardly used in palliative treatment of metastatic disease in every therapeutic line. Unfortunately, the treatment with irinotecan is often associated with severe toxicities, especially neutropenia and diarrhea. The majority of the toxic manifestation is caused by the insufficient deactivation (glucuronidation) of irinotecan active metabolite SN-38 by UGT1A enzyme. The elevated SN-38 plasma concentration is responsible for the hematological and gastrointestinal toxicity that can become life-threatening. The patients carrying the mutation of the gene encoding UGT1A enzyme lack the ability of bilirubin glucuronidation, and suffer from the inherited un-conjugated hyperbilirubinemia (Gilbert syndrome, Crigler-Najjar type 1 and 2 syndrome). The mutations in other enzyme systems also play role in the etiopathogenesis of the irinotecan toxicity: CYP3A (cytochrome P-450), ABC family of transmembrane transporters (adenosine-triphosphate binding cassette). The goal of the contemporary research is to determine the predictive factors that will enable the individual adjustment of the individual drug dosage while minimising the adverse effects and maintaining the treatment benefit.
\end{abstract}

Key words: Irinotecan; SN-38; UGT1A1*28; Pharmacogenetics; Neutropenia; Diarrhea

\section{Introduction}

Colorectal carcinoma is the leading cause of the morbidity and mortality in developed countries. It is responsible for more than $9 \%$ of the overall cancer incidence (46) and stands up as the third most frequent cancer and the fourth most frequent cause of death by natural causes worldwide (14). Approximately 1,200,000 new cases are diagnosed annually (5). The 5 -year survival is reached by only $55 \%$ of the patients in the western countries. The colorectal cancer incidence in Czech Republic in 2009 was 8205 new cases and approximately 7195 patients were treated for the diagnosis in 2010 (9). In 2009 the colorectal carcinoma was the second most frequent cancer both in males and females with decreasing incidence and mortality in comparison with the previous year (42). With the incidence increasing the higher number of patients with metastatic disease undergo the palliative treatment. Although the goal of the therapy, in that case, is not to achieve the complete response but to prolong the survival and improve the quality of life, in a subgroup of patients with liver metastases the combination of chemotherapy with surgical procedure can make the disease curable with 5-year survival exceeding $50 \%$ (1). The first available cytotoxic drug used for the treatment of metastatic colorectal cancer was 5-fluorouracil (5-FU); if administered as a bolus without folinic acid then provided low response rates and limited survival enhancement. The combination with folinic acid and the prolongation of drug infusion have considerably improved the efficiency of single-drug 5-FU. The median survival reached 14 months and more than duplicated the benefit of the best supportive care (24). In addition, the discovery of other active drugs, namely irinotecan and oxaliplatin in 2000 and their combination with 5-FU and folinic acid in FOLFIRI (with irinotecan) and FOLFOX (with oxaliplatin) regime obtained response rates in the range of $40-60 \%$ with an overall survival benefit of more than 20 months $(7,8,41)$. Furthemore, the oxaliplatin found its firm place in the adjuvant setting of the stage II and III colorectal cancer (2) whereas the irinotecan has failed in this indication. There was no statistically significant benefit in both disease-free survival (DFS) and overall survival (OS) after adding irinotecan to infusion/bolus 5-FU/folinic acid in the adjuvant setting in three randomised trials (34, $43,49)$. The evolution of the biological agents set another milestone in the treatment of the metastatic colorectal carcinoma. The backbone of the palliative treatment of the metastatic colorectal cancer is currently formed by the combination of the "classic" chemotherapy based on the fluoropyrimidin derivative (5-fluorouracil, or his pro-drug capecitabine) with folinic acid and irinotecan/oxaliplatin in combination with monoclonal antibody directed against vascular endothelial factor - VEGF (bevacizumab) or epidermal growth factor receptor - EGFR (cetuximab, panitumumab). Irinotecan can be used both as first and second line therapy. The combination of single agent irinotecan 
with EGFR-inhibitor (cetuximab) can be applied after failure of fluoropyrimidin derivative. In addition to colorectal cancer the irinotecan was found to be effective in other solid tumors as well (gastric, lung, cervical, ovarian cancer, neuroendocrine tumours, glioblastoma).

\section{Irinotecan metabolism - introduction}

The metabolic pathways of irinotecan are complex and include the number of enzymes and transporter proteins taking part both in irinotecan activation and deactivation/ elimination. The synthesis of the active irinotecan metabolite $\mathrm{SN}-38$ is encoded by the gene polymorphisms resulting in the distinct interindividual variability in the response to irinotecan administration. It was found out that the gene polymorphisms participating in the irinotecan metabolism can lead to the limiting toxicity (grade 3 and 4 neutropenia, severe late-onset diarrhea) and the dose reduction or even cessation of the chemotherapy. This can result either in the life-threatening treatment related morbidity or the decrease of the relative dose intensity that reduces the treatment benefit and the chances for the cure or the prolongation of the life. The understanding of the mechanism of the irinotecan action, the survey of its clinically relevant genetic variations and enabling the screening of these variations that would be both quick and financially acceptable before the treatment onset in the scope of the tailored treatment can lead to the individual dose adjustments according to the personal genetic profile while maintaining the treatment efficacy and lowering the risk of the toxicity.

\section{Metabolic pathways of irinotecan - overview}

Irinotecan $\{$ CPT-11, 7-ethyl-10[4-(1-piperidino)-1-piperidino]carbonyloxycamptothecin $\}$ is the semisynthetic analog of the natural alkaloid camptothecin. Irinotecan; the pro-drug, is hydrolysed into its active metabolite SN-38 by carboxylesterases ( 2 isoforms CES1, CES2, present in plasma, intestinal and tumour tissue and in high content in the liver). SN-38 is $100-1000$ times more cytotoxic than the parent compound, bounds to and inhibits the DNA-topoisomerase-I complex - the nuclear enzyme that relaxes torsionally strained DNA (supercoiled) during the course of the DNA replication (S-phase). The blockage results in single-strand DNA breaks that are converted to double-strand breaks and programmed cell death occurs. SN-38 is subsequently glucuronidated in the liver by uridine diphosphate glucuronosyltransferase UGT1A1 to form an inactive metabolite, SN-38G. Besides the activity of UGT1A1 the other liver enzyme UGT1A9 and extrahepatic enzyme UGT1A7 participate in the deactivation of SN-38 (13). The second metabolic pathway of irinotecan deactivation is its oxidation by cytochrome P450 3A, the member of cytochrome P450 enzymes superfamily, resulting in the formation of inactive metabolites APC (\{7-ethyl-10[4-N-(5-aminopentanoicacid)-1-piperidino] carbonyloxycamptothecin $\})$ and NPC ( $\{7$-ethyl-10[4-amino-1-piperidino]carbonyloxycamptothecin $\})$. NPC metabolite can further increase the SN-38G plasma levels by the carboxylesterases mediated hydrolysis. Inactive SN-38G is excreted via bile duct into the small intestine and cleaved by bacterial endogenous $\beta$-glucuronidases in the colon to regenerate $\mathrm{SN}-38$ that is subsequently responsible for the direct intestinal mucosa injury and the late-onset diarrhea occuring approximately 5-7 days after irinotecan administration and affecting about $40 \%$ of the patients $(6,30)$.

\section{Plasma disposition}

In blood, $80 \%$ of irinotecan (CPT-11) is mainly bound to erythrocytes, whereas SN-38 is bound for at least $99 \%$ to albumin and lymphocytes (also to erythrocytes and neutrophils). Both irinotecan (CPT-11) and SN-38 are present in two distinguishable forms, an active lactone ring form and an inactive carboxylate form, between which a $\mathrm{pH}$-dependent equilibrium exists. The lactone species is predominantly formed in the acid $\mathrm{pH}$ whereas the basic $\mathrm{pH}$ favors formation of the carboxylate form. The antitumour effect is solely mediated by the lactone form that is essential for interaction with the DNA-enzyme complex. SN-38 lactone form also binds significantly stronger to albumin than the corresponding carboxylate form which explains the better stability of SN-38 in vivo compared to CPT-11 that does not show any difference in binding to albumin between its two forms.

\section{Metabolic pathways of irinotecan - carboxylesterases, CYP3A, ABC}

Irinotecan is hydrolysed by carboxylesterases into its active metabolite SN-38. Carboxylesterasis (CES) is present in plasma, intestinal tissue (enterocytes), tumor cells and especially in the hepatocytes. The recent data suggest that intra-tumoral activation of irinotecan by CES might be more important than its hepatic and systemic activation (48). CES exists in 2 isoforms, CES1 and CES2. In vitro studies, the activity of CES2 enzyme is found much more potent than CES1 in the irinotecan activation (35). Although several CES gene polymorphisms have been identified confirming the interpatient variation in CES activity there are currently no data evaluating their significance for the rate of SN-38 formation from irinotecan. Irinotecan is also oxidized by cytochrome P450 3A4 and 3A5, the members of the superfamily of cytochrome P450 enzymes. Especially CYP3A4 seems to be of particular importance whereas CYP345 is expressed to a lesser extent in most Caucasians (25). Irinotecan is converted via this pathway into APC and NPC (see above). NPC can be further activated by CES into SN-38 and thereby can indirectly increase its plasma concentration. Several polymorphisms have been found in the CYP3A gene related to the functional enzyme alteration but the contemporary research suggests these variations to 
be predominantly influenced more-likely by environmental and physiologic factors (concomitant medications, nutrition, alteration of liver function, patient's performance status) than by genetic factors $(10,27,29)$. Because the large number of all drugs is metabolised via CYP3A the commonly co-administered medication is able to influence its activity and irinotecan/SN-38 plasma concentrations in the next step just as well. Ketoconazole, the classic CYP3A inhibitor, blocks the irinotecan conversion into APC and NPC almost completely. The frequently used drugs in combination with the irinotecan-based chemotherapy loperamid and ondansetron decrease the APC and NPC formation for more than $50 \%$ (loperamide) and $25-75 \%$ (ondansetron) (14). This mechanism leads to the increased formation of SN-38 because of the advanced irinotecan offer and thus to the toxicity and possibly the therapeutic effect potentiation. Cyclosporine increases the AUC of SN-38 by $23 \%$ to $630 \%$ through the inhibition of P-glycoprotein mediated biliary secretion. In contrast, enzyme-inducing anticonvulsants, phenytoin, phenobarbital and carbamazepine, increase the clearance of irinotecan (CPT-11) and cause a decrease in systemic exposure to the active metabolite SN-38. At ASCO meeting in 2010 was presented the study investigating the impact of the co-administered statins in combination with irinotecan infusion every 3 weeks in the group of 33 patients (26 males, 7 patients treated with statins). The patients on statin therapy showed the statistically significantly lower ANC nadir (absolute neutrofil count, $\mathrm{p}=0.001$ ), higher irinotecan AUC (area under the concentration-time curve, $\mathrm{p}<0.0001)$ and higher SN-38 AUC $(\mathrm{p}=0.005)$. However, the impact on the treatment effect was not discovered (19). Also the adenosine-triphosphate binding cassette (ABC) transporters are the large family of transmembrane proteins that play important role in the irinotecan absorption and excretion. The most important proteins are 1) P-glycoprotein (MDR1, ABCB1), 2) the canalicular multispecific organic anion transporter (c-MOAT, MRP2, ABCC2), and 3 ) the breast cancer resistance protein (BCRP, ABCG2) (29). The membrane transporters are responsible for the SN-38 absorption from plasma into the hepatocyte, excretion of irinotecan and its metabolites into the bile and the SN-38 efflux from hepatocyte into the interstitium. Recent studies have revealed a number of polymorphisms in the genes encoding these transporters. More than 50 single-nucleotide polymorphisms were detected in P-glycoprotein gene alone. At ASCO meeting in 2010 was presented the study investigating the impact of the functional ABCB1 variants on the irinotecan toxicity in 115 patients with metastatic colorectal cancer. The ABCB1 $1236 \mathrm{C}>\mathrm{T}$ polymorphism was the predictor of severe late-onset diarrhea and better clinical response in this study (25). The detection of this polymorphism seems promising in the common haplotype $-1236 \mathrm{C}>\mathrm{T}+2677 \mathrm{G}>\mathrm{A} / \mathrm{T}+3435 \mathrm{C}>\mathrm{T}$. Furthemore, the haplotype ABCC2-24C $>\mathrm{T}+3972 \mathrm{C}>\mathrm{T}$ seems to be potentially useful as the secondary screening in patients lacking UGT1A1*28 mutation.

\section{Metabolic pathways of irinotecan - detoxification of $\mathrm{SN}-38$ by UGT1A}

The microsomal uridine diphosphate-glucuronosyltransferase (UGT) is the membrane phase II enzym ensuring the detoxification of a range of endogenous (bilirubin, estrogen e.c.) and exogenous (lipophilic) substrates by changing hydrophobic molecules into solubile derivatives that are subsequently excreted in urine and through bile in feces. In urine, CPT-11 and SN-38G are the main compounds with CPT-11 accounting for about $10-20 \%$ of the administered dose. Fecal excretion is the major route of drug elimination, with $52-64 \%$ of the drug recovered. The unexpectedly high SN-38 concentrations and relatively low $\mathrm{SN}-38 \mathrm{G}$ concentrations in fecal specimen are sugestive for substantial $\beta$-glucuronidase activity in human intestinal content. These processes are induced by the transfer of a glucuronic acid from uridine diphosphoglucuronic acid to the given compound. The human UGT superfamily has been classified into the UGT1A, UGT2A and UGT2B subfamilies. The most clinically important UGT1A subfamily is encoded by a single gene locus on chromosome $2 \mathrm{q} 37$ (44) and especially the isoforms 1A1, 1A7 and 1A9 are involved in the phase II conjugation of SN-38 to the inactive metabolite SN-38G. UGT1A1 and UGT1A9 are highly expressed in the gastrointestinal tract and the liver whereas UGT1A7 is only expressed in extrahepatic tissues (esophagus, stomach and lung) and its role remains unclear $(3,22,45)$. The human UGT1A gene complex consists of nine active and four inactive exon 1 segments and common exons 2-5. Each UGT1A gene transcript is formed by splicing one of the first exons with the common exons $2-5$. The function of UGT1A is the conjugation of SN-38 with glucuronic acid to form the inactive metabolite SN-38G that can be easily excreted via bile into the feces where it undergoes the deglucuronidation by the bacterial $\beta$-glucuronidases present in the colon. The re-generated active SN-38 is re-absorbed via the enterohepatic recirculation while damaging directly the intestinal mucosa at the same time and becomes the cause of the late-onset diarrhea 5-7 days after the irinotecan infusion $(6,30)$. The most explored isoform so far is UGT1A1 with more than 110 functional variants reported. The prevalence of the homozygotic form of the most common polymorphism UGT1A1*28 is $10 \%$ in western countries. This polymorphism resulting in absent or very low UGT1A activity has been associated with three inherited unconjugated hyperbilirubinemia syndromes: Crigler-Najjar syndrome type 1 and 2, and Gilbert's syndrome that differ only in the UGT1A1 activity and the bilirubin plasma level (Gilbert's syndrome is the mildest, Crigler-Najjar syndrome type 1 the most intense type) (38). The mentioned genetic variant of UGT1A1 occurs in the TATA promoter region and has variable repeats of thyminadenine (TA) dinucleotides. The wild-type promotor $(\mathrm{UGT} 1 \mathrm{~A} 1 * 1)$ has six TA repeats on each allele whereas the 
most frequent polymorphism UGT1A1*28 seven, therefore this polymorphism is also represented as UGT1A $1 * 28_{7 / 7}$, in which the figure ${ }_{7 / 7}$ represents the number of TA repeats on each allele and means the homozygosity (the heterozygosity is thus labeled as UGT1A $1 * 28_{6 / 7}$ ). An increase in dinucleotide repeats within the TATA region leeds to a considerable reduced enzyme expression of about 30-80\% (41) and the UGT1A1 activity appears to be inversely related to the number of TA repeats. The frequency of the TA repeats has been reported to vary among ethnic populations. The less frequent variant alleles $(\mathrm{TA})_{5}(=\mathrm{UGT} 1 \mathrm{~A} 1 * 36)$ and $(\mathrm{TA})_{8}$ (=UGT1A1*38) have been identified mainly in the African population. In Asia the UGT1A1*28 frequency is low, the most common polymorphism is UGT1A1*6 (single-nucleotid polymorphism in exon 1 ), that occurs in $18-23 \%$ of the population $(32,39)$.

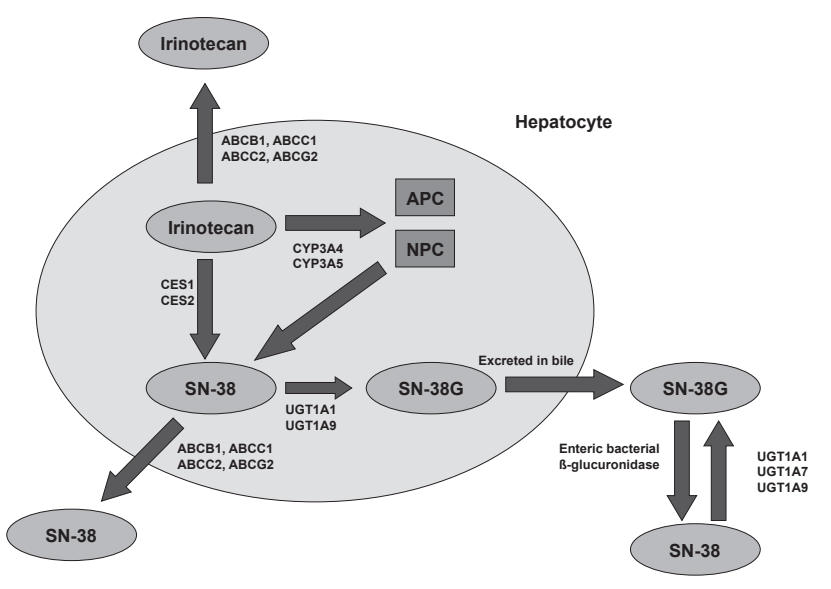

Fig. 1: Metabolic pathways of irinotecan - scheme (16)

\section{Irinotecan toxicity and its assessment}

The treatment with irinotecan is associated with the plentiful profile of toxicity. Besides the most frequent and clinically important myelosuppresion and diarrhea the other frequent side effects can occur: nausea and vomiting, dyspnea, maculopapular eruption and excessive sweating, stomatitis, alopecia, asthenia and transient elevation of serum transaminases, alkaline phosphatase and bilirubin. Neutropenia and diarrhea are the most frequent adverse events of the irinotecan administration and the ones that can jeopardize the patient's life. Life-threatening diarrhea occurs in $25 \%$ of the patients. The etiology of the diarrhea falls into two categories: 1 . The early-onset diarrhea originated during the drug infusion or not later than six hours after the irinotecan administration, cholinergic-related, and can be prevented or treated with atropine, 2 . The late-onset diarrhea with the onset usually later than 24 hours after the irinotecan administration caused by the SN-38G deglucuronidation via the bacterial $\beta$-glucuronidases in the colon, the re-generated SN-38 subsequently affects the intestinal mucosa. This type of diarrhea is treated with loperamide. Among the various pharmacokinetic parameters SN-38 AUC indicates the strongest correlation with the hematological and gastrointestinal toxicity. Recently, the several murine studies have reported the efficacy of selective $\beta$-glucuronidases inhibitors in aleviating the SN-38 related gastrointestinal toxicity. These inhibitors are targeted against the sequences specific for the bacterial enzyme, therefore they do not affect the human cells nor the bacteria of the physiological microflora whose integrity is important especially in the patients with the altered immunity (following the chemotherapy or radiotherapy) (6). The incidence of life-threatening neutropenia is observed in approximately $35 \%$ of the patiens undergoing irinotecan therapy. It has been proved that the correlation between the presence of UGT1A $1 * 28$ mutation and the toxicity (both heamatological and gastrointestinal) is significant only at intermediate and high doses. The UGT1A $1 * 28_{7 / 7}$ patients have 27.8 fold higher risk of developing grade 4 neutropenia than the patients with UGT1A $1 * 28_{6 / 7}$ or UGT1A $1 * 28_{6 / 6}$ genotypes $(95 \%$ confidence interval $4.0-195, \mathrm{p}<0.005)$ after administration of intermediate and high doses of irinotecan (i.e. $200-350 \mathrm{mg} / \mathrm{m}^{2}$ ) (17). The largest meta-analysis in 1760 patients assessed the risk of developing the severe diarrhea 3.69-fold higher in homozygous genotype UGT1A $1 * 28_{7 / 7}$ than in UGT1A $1 * 28_{6 / 6}$ patients $(95 \%$ confidence interval $2.06-6.83, \mathrm{p}<0.001)$ whereas the patients with UGT1A $1 * 28_{6 / 7}$ heterozygous genotype have the risk 1.92 -fold higher in comparison with UGT1A $1 * 28_{6 / 6}(95 \%$ confidence interval 1.31-2.82, $\mathrm{p}<0.001)$. Similarly, no association was found between UGT1A $1 * 28$ expression and the severe diarrhea at doses lower than $125 \mathrm{mg} / \mathrm{m}^{2}(50)$. The UGT1A1 gene is naturally the target for the number of other polymorphisms. It was detected that the severe neutropenia occurs much more frequently in patients with UGT1A $1 * 28+-3156 \mathrm{G}>\mathrm{A}$ haplotype than in those without $-3156 \mathrm{G}>\mathrm{A}$ polymorphism (21). Additionally, UGT1A7 and UGT1A9 also play their role in SN-38 glucuronidation. For example, UGT1A7*3 is associated with haematological toxicity and UGT1A7*2, UGT1A7*3, UGT1A9-118 with the tumor response to irinotecan. Another possibility and unlike earlier mentioned genetic testing easily usable in the common clinical practise might be the assessment of the pre-treatment total bilirubin level as the predictor of irinotecan toxicity. Bilirubin is an endogenous substrate for UGT1A1, and it has been suggested that the patient bilirubin level could serve as an inexpensive surrogate marker of UGT1A1 status. Several studies have demonstrated strong relation between the pre-treatment total bilirubin levels and therapy-induced grade 3 and 4 neutropenia. Fujiwara et al. have proved 4.9 -fold higher risk of grade 4 neutropenia in the patients with lung cancer treated with cisplatin (60 or $\left.80 \mathrm{mg} / \mathrm{m}^{2}\right)$ on day 1 and irinotecan $\left(60 \mathrm{mg} / \mathrm{m}^{2}\right)$ on day 1 and 8 every 3 weeks (odds ratio 4.9, 95\% confidence interval 1.4-17.7) with the median pre-treatment total bilirubin 
$0.7 \mathrm{mg} / \mathrm{dl}$ compared to the patients with $0.5 \mathrm{mg} / \mathrm{dl}$ level $(p=0.03)$. Interestingly, this relation was not significant for the diarrhea at the same time. The diarrhea occurence was found to be associated only with the presence of liver metastases, high cisplatin dose and low pre-treatment neutrofil count (12). In Kramar study embodying 49 patients with the metastatic colorectal cancer treated with single irinotecan $350-500 \mathrm{mg} / \mathrm{m}^{2}$ every 3 weeks in the first line the grade 3 or 4 neutropenia was observed in $50 \%$ of treatment cycles in patients with bilirubin level higher than $1.5 \mathrm{mg} / \mathrm{dl}$ and in $17 \%$ with the level lower than $0.4 \mathrm{mg} / \mathrm{dl}$ $(\mathrm{p}=0.01)$. In this study the bilirubin was evaluated before each treatment cycle and was associated with the occurence of haematological toxicity during the following cycle. Again, the relation to diarrhea was not found (23). Innocenti et al. have detected the significant correlation between grade 3 and 4 neutropenia and bilirubin levels in range $1.0-1.5 \mathrm{mg} / \mathrm{dl}(\mathrm{p}=0.03)$ in 287 patients treated with single-agent irinotecan every 1 or 3 weeks, albeit the correlation was significant only in the weekly regime. The initial bilirubin level failed to predict any other toxicity or efficacy (21). In contrast to this data, Parodie et al. have not proved the impact of initial bilirubin concentration on the nadir value of the absolute neutrofil count in patients treated for the metastatic colorectal cancer with irinotecan in the first line. The impact remained insignificant even when the age, gender and UGT1A1 genoytpe were added to the predictive model (33). Because of the inter-study discrepancies mentioned above there are currently no official guidelines available that would recommend the irinotecan individual dose adjustments or profylactic G-CSF application according to the pre-treatment bilirubin levels for the clinical practise. It is mainly due to the fact that the bilirubin level is influenced by various factors and not only by UGT1A status alone. The increase of bilirubin can occur in the presence of liver metastases, direct liver damage caused by the cytotoxic drugs, thrombosis of liver veins with subsequent modification of hepatobiliary tree, the use of protease inhibitors etc. Since the urinary excretion of unchanged irinotecan (CPT-11) and $\mathrm{SN}-38 \mathrm{G}$ plays the significant role in the elimination of the drug the impact of the altered renal function on the irinotecan toxicity has been evaluated. De Jong et al. found the four-times higher risk of grade 3-4 neutropenia in 131 patients with slower creatinine clearance $(35-66 \mathrm{ml} / \mathrm{min})$ treated with irinotecan in a three-weekly schedule (58\% vs. $14 \% ; \mathrm{p}<0.001)(11)$. Non-genetic factors such as age, gender, ethnicity, body surface area, height, and weight failed in predicting the toxicity.

\section{Current clinical practise and future perspectives}

In July 2005 FDA (Food and Drug Administration) with Pfizer Pharmaceuticals changed the package insert for the pharmacogenetic testing of UGT1A1*28 in irinotecan-treated patiens recommending to reduce the irinotecan dose in UGT1A $1 * 28$ carriers. This was stimulated by four pharmacogenetic studies that managed to identify 2.5-17.0-fold higher risk of developing the toxicity in patiens treated with irinotecan and carrying UGT1A $1 * 28$ mutation. However, it remains evident that the testing should be carried out only at intermediate and high irinotecan doses (120-200 mg/m²). At low doses UGT1A1*28 status failed to predict both haematological and gastrointestinal toxicity. Analysing the studies, the positive and negative predictive value of UGT1A $1 * 28$ was determined 0.53 and 0.87 , respectively, which means that $53 \%$ of UGT1A $1 * 28$ carriers will develop the grade 3-4 neutropenia in comparison with $13 \%$ of patiens with UGT1A $1 * 28_{6 / 6}$ genotype. Thus, the UGT1A1 mutational status is certainly not the only clinical predictor of toxicity. The metabolism of irinotecan and its active metabolite SN-38 is influenced by the mutations in genes encoding other enzymes as well. The most promising option is the assessment of polymorphisms in transmembrane protein genes - ABCB1, ABCC1-2 and ABCG2 whereas the recent studies have registered the best toxicity predictions by detecting these polymorphisms within the specific haplotypes. The non-genetic factors (such as diet, concomitant diseases, performance status and medication, demographic factors etc.) can also affect the activity of cytochrome P450 superfamily enzymes (especially CYP3A4 and CYP3A5 isoforms) and have the undeniable impact on irinotecan metabolism. It has been proved, for example, that the smokers exhibit significantly lower irinotecan AUC and about, 40\% lower SN-38 exposure and higher degree of SN-38 glucuronidation in comparison to the non-smokers. On other hand, green tea catechines block the UGT1A1-catalysed glucuronidation which leads to the decrease of SN-38G plasma concentration (i.e. to the increase of SN-38 level) resulting in the toxicity potentiation. Simultaneously, the catechines inhibit the CYP3A4-dependent irinotecan oxidation in liver microsomes which lowers NPC and consecutively SN-38. Apparently, the environmental factors seem to influence the CYP450 enzyme group in a very heterogenous and complex way, hence it is no surprise that the pharmacogenetic testing of any of its isoforms has not shown the clinical benefit yet (31). Identically, no mutational genotype status of enzyme carboxylesterases 1 and 2 (i.e. CES1, CES2 isoforms) has proved the usefulness in predicting the toxicity. The goal of ongoing research is to find the predictive factors of irinotecan toxicity that could get implemented into the common clinical practise and by which we would be able to adjust the irinotecan dose according to the genetic and non-genetic factors of the particular patient. The assessment of UGT1A1*28 and the transmembrane transporter ABC haplotype status in combination with non-genetic environmental factors (performance status, liver metastases, concomitant medication, cytotoxic therapy, pre-treatment bilirubin level, pre-treatment neutrofil count etc.) before the administration of the first dose of irinotecan seems to be the perspective 
approach. After finding the eligible combination of these predictive factors that would most reliably predict the potential toxicity the patient could be offered the individual-tailored therapy corresponding to his specific genotype, tumor characteristics, other diseases and environmental factors. The result will be the reduction of chemotherapy-related toxicity while maintaining the therapeutical benefit, prolongating the overall survival, improving the quality of life and last but not least reducing the toxicity-related financial costs (antibiotics, G-CSF).

\section{Acknowledgements} $36 / 07$

This review report was supported by the PRVOUK

\section{References}

1. Adam R, Delvart V, Pascal G et al. Rescue surgery for unresectable colorectal liver metastases downstaged by chemotherapy: a model to predict long-term survival. Ann Surg 2004; 240: 644-57.

2. André T, Boni C, Navarro $M$ et al. Improved overall survival with oxaliplatin, fluorouracil and leucovorin as adjuvant treatment in stage II or III colon cancer in the MOSAIC trial. Journal of Clinical Oncology 2009; 27: 3109-16.

3. Barbier O, Girarad H, Inoue $\mathrm{Y}$ et al. Hepatic expression of the UGT1A9 gene is governed by hepatocyte nuclear factor 4alpha. Mol Pharmacol 2005; 67: 241-49.

4. Beutler E, Gelbart T, Demina A. Racial variability in the UDP-glucuronosyltransferase 1 (UGT1A1) promoter: a balanced polymorphism for regulation of bilirubin metabolism? Proc Natl Acad Sci USA 1998 Jul 7; 95(14): 8170-74.

5. Boyle P, Langman J S. ABC of colorectal cancer: Epidemiology. BMJ 2000; 321(7264): 805-8.

6. Bret D Wallace, Hongwei Wang, Kimberley T Lane et al. Alleviating Cancer Drug Toxicity by Inhibiting a Bacterial Enzyme. Science 2010 Nov 5; Vol. 330, no. 6005: 831-5.

7. De Gramont A, Figer A, Seymour M et al. Leucovorin and fluorouracil wit or without oxaliplatin as first-line treatment in advanced colorectal cancer. Journa of Clinical Oncology 2000; 18: 2938-47.

8. Douillard JY, Cunningham D, Roth AD et al. Irinotecan combined with fluorouracil compared with fluorouracil alone as first-line treatment for metastatic colorectal cancer: a multicentre randomised trial. Lancet 2000; 355: 1041-1047.

9. Dušek L, Mužík J, Babjuk M et al. Populační odhady počtu nemocných s kolorektálním karcinomem v ČR - jeden z nástrojů hodnocení včasné diagnostiky časných stadií a rekurence onemocnění. Farmakoterapie 2009; 5: 11-20.

10. Floris A de Jong, Maja JA de Jonge, Jaap Verweij, Ron HJ Mathijssen. Role of pharmacogenetics in irinotecan therapy. Cancer Letters 2006; 234: 90-106.

11. Floris A de Jong, van der Bol JM, Mathijssen RH et al. Renal function as a predictor of irinotecan-induced neutropenia. Clin Pharmacol Ther. 2008 Aug; 84(2) 254-62.

12. Fujiwara Y, Sekine I, Ohe Y et al. Serum total bilirubin as a predictive factor for severe neutropenia in lung cancer patients treated with cisplatin and irinotecan. Jpn J Clin Oncol 2007; 37(5): 358-64.

13. Gagne JF, Montminy V, Belanger P, Journault K, Gaucher G, Guillemette C et al. Common human UGT1A polymorphisms and the altered metabolism of irinotecan active metabolite 7-ethyl-10-hydroxycamptothecin (SN-38). Mol Pharmacol 2002; 62: 608-17.

14. Garcia J, Jemal A, Ward EM et al. Global cancer facts and figures 2007, Atlanta, GA: American cancer society, 2007.

15. Haaz M-C, Riché C, Rivory LP, Robert J. Biosynthesis of an aminopiperidino metabolite of irinotecan [7-ethyl-10-[4-(1-piperidino] carbonyloxycamptothecine] by human hepatic microsomes. Drug Metabolism and Disposition 1998; 26: 769-74.

16. Scheme adjusted according to Hahn K, Wolff J, Kolesa J. Pharmacogenetics and Irinotecan Therapy. American Journal of Health-System Pharmacy. 2006; 63(22): 2211-17.

17. Hoskins JM, Goldberg RM, Qu P, Ibrahim JG, McLeod HL. UGT1A1*28 genotype and irinotecan-induced neutropenia: dose matters. J Natl Cancer Inst 2007; 99: $1290-5$.
18. Charasson V, Bellott R, Meynard D, Longy M, Gorry P, Robert J. Pharmacogenetics of human carboxylesterase 2, an enzyme involved in the activation of irinotecan into SN-38. Clin Pharmacol Ther 2004; 76: 528-35.

19. Innocenti F, Karrison T, Ramirez J et al. Effect of coadministration of statins on neutropenia in a phase I genotype-directed dose-escalation study of irinotecan. Journal of Clinical Oncology 2010; 28(suppl May 20): 3020.

20. Innocenti F, Ratain MJ. Irinotecan treatments in cancer patients with UGT1A1 polymorphisms. Oncology 2003; 17(suppl 5): 52-5.

21. Innocenti F, Undevia SF, Iyer L et al. Genetic variants in the UDP-glucuronosyltransferase $1 \mathrm{~A} 1$ gene predict the risk of severe neutropenia of irinotecan. Journal of Clinical Oncology 2004; 22(8): 1382-8.

22. Jinno H, Saeki M, Saito Y et al. Functional characterization of human UDP-glucuronosyltransferase $1 \mathrm{~A} 9$ variant, D256N, found in Japanese cancer patients. J Pharmacol Exp Ther 2003; 306: 688-93.

23. Kramar A, Gourgou-Bourgade S, Ychou M. Relationship of serum bilirubin to toxicity in patiens with metastatic colorectal cancer treated with single-agent high-dose irinotecan. Journal of Clinical Oncology 2004; 23(3): 650.

24. Labianca R, Pessi MA, Zamparelli G. Treatment of colorectal cancer. Current guidelines and future prospects for drug therapy. Drugs 1997; 53: 593-607.

25. Levesque E, Bélanger A, Coutore F et al. The contribution of UGT1A and $\mathrm{ABCB} 1$ to irinotecan-induced toxicity: A prospective pharmacogenetic study of patients with metastatic colorectal cancer. J Clin Oncol 2010; 28(suppl, abstr 3101).

26. Lin YS, Dowling AL, Quigley SD, Farin FM, Zhang J, Lamba J. Co-regulation of CYP3A4 and CYP3A5 and contribution to hepatic and intestinal midazolam metabolism. Mol Pharmacol 2002; 62: 162-72.

27. Marsh S, Xiao M, Yu J et al. Pharmacogenomic assesment of carboxylesterases 1 and 2. Genomics 2004; 84: 661-8

28. Mathijssen RH, de Jong FA, van Schaik RH et al. Prediction of irinotecan pharmacokinetics by use of cytochrome P4503A4 phenotyping probes. J Natl Cancer Inst 2004; 96: 1585-92.

29. Mathijssen RH, van Alphen RJ, Verweij J et al. Clinical pharmacokinetics and metabolism of irinotecan (CPT-11). Clin Cancer Res 2001; 7: 2182-94.

30. Michael M, Brittain M, Nagai J et al. Phase II study of activated charcoal to prevent irinotecan-induced diarrhea. J Clin Oncol 2004; 22: 4410-7.

31. Mirkov S, Komorski BJ, Ramirez J et al. Effects of green tea compounds on irinotecan metabolism. Drug Metabolism and Disposition 2007; 35 $228-33$

32. Onoue M, Terada T, Kobayashi M et al. UGT1A1*6 polymorphism is most predictive of severe neutropenia induced by irinotecan in Japanese cancer patiens. International Journal of Clinical Oncology 2009; 14: 136-42.

33. Parodi L, Pickering E, Cisar LA, Lee D, Soufi-Mahjoubi R. Utility of pretreatment bilirubin level and UGT1A1 polymorphisms in multivariate predictive model sof neutropenia associated with irinotecan treatment in previously untreated patiens with colorectal cancer. Archives of Drug Information 2008; 1 : $97-106$

34. Saltz LB, Niedzwiecki D, Hollis D et al. Irinotecan fluorouracil plus leucovorin is not superior to fluorouracil plus leucovorin alone as adjuvant treatment for stage III colon cancer: Results of CALGB 89803. Journal of Clinical Oncology 2007; 25: $3456-61$

35. Senter PD, Beam KS, Mixan B, Wahl AF. Identification and activities of human carboxylesterases for the activation of CPT-11, a clinically approved anticancer drug. Bioconjug Chem 2001; 12: 1074-80.

36. Spareeboom A, Danesi R, Ando Y, Chan J, Figg WD. Pharmacogenomics of ABC transporters and its role in cancer chemotherapy. Drug Resisct Updat 2003; 6: $71-84$.

37. Strassburg CP. Gilbert-Meulengrachts's syndrome and pharmacogenetics: is jaundice just the tip of iceberg? Drug Metab Rev 2010; 42: 162-75.

38. Strassburg CP. Hyperbilirubinemia syndromes (Gilbert-Meulengracht, Crigler-Najar, Dubin-Johnson and Rotor syndrome). Best Practise \& Research Clinical Gastroenterology 2010; 24: 555-71.

39. Takeuchi K, Kobayashi Y, Tamaki S et al. Genetic polymorphisms of bilirubin uridine diphosphate-glucuronosyltransferase gen in Japanese patiens with Crigler-Najar syndrome or Gilbert's syndrome as well as in healthy Japanese subjects. J Gastroenterol Hepatol 2004; 19: 1023-8.

40. Thummel KE, Lamba JK, Lin YS, Schuetz EG. Genetic contribution to viable human CYP3A-mediated metabolism. Adv Drug Deliv Rev 2002; 54: 1271-94.

41. Tournigand C, André T, Achille E et al. FOLFIRI followed by FOLFOX6 or the reverse sequence in advanced colorectal cancer: a randomized GERCOR study. Journal of Clinical Oncology 2004; 22(2): 229-37.

42. ÚZIS ČR, Aktuální informace, Zhoubné nádory v roce 2009 (released 24/01/2012). (Accessed at www.uzis.cz/system/files/02_12.pdf.)

43. Van Cutsem E, Labianca R, Bodoky G et al. Randomized phase III trial comparing biweekly infusional fluorouracil/leucovorin alone or with irinotecan in the adjuvant treatment of stage III colon cancer: PETACC-3. Journal of Clinical Oncology 2009; 27: 3117-25. 
44. Van Es HH, Bout A, Liu J et al. Assignment of the human UDP glucuronosyl-transferase gene (UGT1A1) to chromosome region 2q37. Cytogenet Cell Genet 1993; 63: 114-6.

45. Vogel A, Kneip S, Barut A et al. Genetic link of hepatocellular carcinoma with polymorphisms of the UDP-glucuronosyl-transferase UGT1A7 gene. Gastroenterology 2001; 121: 1136-44.

46. World Cancer Research Fund and American Institute for Cancer Research Food, Nutrition, Physical Activity, and the Prevention of Cancer: A Global Perspective. 1st ed. Washington DC. American Institute for Cancer Research, 2007.

47. Wu MH, Chen $\mathrm{P}, \mathrm{Wu} \mathrm{X}$ et al. Determination and analysis of single nucleotide polymorphisms and haplotype structure of the human carboxylesterase 2 gene. Pharmacogenetics 2004; 14: 595-605.

48. Wu MH, Yan B, Humerickhouse R, Dolan ME. Irinotecan activation by human carboxylesterases in colorectal adenocarcinoma cells. Clin Cancer Res 2002; 8: 2696-2700.

49. Ychou M, Raoul JL, Douilliard JY et al. A phase III randomised trial of LV5FU2 + irinotecan versus LV5FU2 alone in adjuvant high-risk colon cancer (FNCLCC Accord02/FFCD9802). Ann Oncol 2009; 674-80.

50. Zhe-Yi Hu, Qi Yu, Yuan-Sheng Zhao. Dose-dependent association between UGT1A1*28 polymorphism and irinotecan-induced diarrhoea: a meta-analysis. European journal of cancer 2010; 46: 1856-65.

Received: $13 / 02 / 2012$

Accepted in revised form: 03/11/2012

\section{Corresponding author:}

Adam Paulík, M.D., University Hospital Hradec Králové, Department of Oncology and Radiotherapy, Sokolská 581, 50005 Hradec Králové, Czech Republic; e-mail: a.paulik@seznam.cz 